\title{
Modeling of bicomponent mixing system used in the manufacture of wind generator blades
}

\author{
Esteban Jove ${ }^{1,2}$, Héctor Aláiz-Moretón ${ }^{2}$, José Luis Casteleiro-Roca ${ }^{1}$, Emilio \\ Corchado $^{3}$, and José Luis Calvo-Rolle ${ }^{1}$ \\ 1 Departamento de Ingeniería Industrial, \\ Escuela Universitaria Politécnica, \\ University of A Coruña, \\ Avda. 19 de febrero s/n, 15495, Ferrol, A Coruña, Spain \\ esteban.jove@udc.es \\ 2 Departamento de Ingeniería Eléctrica y de Sistemas y Automática, \\ Escuela de Ingenierías Industrial e Informática, \\ Universidad de León, \\ Campus de Vegazana s/n, 24071, León, León, España \\ 3 Departamento de Informática y Automática, \\ Universidad de Salamanca, \\ Plaza de la Merced s/n, 37008, Salamanca, Salamanca, España
}

\begin{abstract}
The clean energy use has increased during the last years, especially, electricity generation through wind energy. Wind generator blades are usually made by bicomponent mixing machines. With the aim to predict the behavior of this type of manufacturing systems, it has been developed a model that allows to know the performance of a real bicomponent mixing equipment. The novel approach has been obtained by using clustering combined with regression techniques with a dataset obtained during the system operation. Finally, the created model has been tested with very satisfactory results.
\end{abstract}

Keywords: Clustering, SOM, MLP, SVM, Wind generator

\section{Introduction}

There was an increase of the energy consumption and a raise of the enviromental pollution during the last years. This fact involves the expansion of the renewable energy generation [30].

In this context, wind energy solutions has increased over the world. With the aim to improve the competitive all the involved tasks have been and still being improved [17]. One of these improvements is the use of carbon fiber as a base material to manufacture wind generator blades. This compound is obtained by mixing components [28]. They are called bicomponents because these materials have different characteristics when they are separated. However, they react when they are mixed and their properties change significantly. 
In the system studied on the present research, one of the components is an epoxy resin, and the order one is a catalyst. Both components are NonNewtonian fluids, which means that their mechanical properties do not remain constant with variation of efforts received [6]. On this study, a $1: 1$ ratio is required in the final compound to obtain the best properties. The obtained material with the above mentioned components has the advantages of high specific strength and stress, then, it is suitable for the use in wind generator blades [28].

The installation studied is a mixing machine, which have two reservoirs, one by each component, epoxy resin and catalyst. Each tank feeds one pump, and both have to deliver the same flow rate to obtain a constant ratio in the mixing valve.

Due to the characteristics of the fluid and the variation of the pump efficiency, it is a trouble to obtain the needed ratio for the final material. The purpose of this study is to create a model with the aim to predict the behavior of the system. It is obtained by data processing of the information obtained over the real installation when it is in normal operation. Thus, by modeling the system it is possible to predict the flow rate, the ratio and other system variables.

It is possible to split the layout of predictive models in two methods [18]:

- Global models: The model is generated from all available data for training, trying to get the lowest error. Different techniques can be used to generate the model: Multi-Layer Perceptron (Articial Neural Networks, ANN) [2,3, 1] and Support Vector Regression (SVR) [12].

- Local models: all the dataset is divided into different clusters, depending on the characteristics of the input data. Typically, in order to make the groups, it can be used algorithms like Self Organization Map (SOM) $[4,8]$ or K-means $[10,11]$. After that, regression techniques are applied for each cluster.

This paper is organized as follows. It begins with a short description of the case study followed by an explanation of the models to describe the system behaviour. In the next section, results are presented, and finally the conclusions and the acknowledgments are shown.

\section{Case of study}

\subsection{Mixing machine installation}

Before system behavior modeling, it is necessary to know the parts of the installation and its operation. As was mentioned in the introduction section, the system aim is mix two components on the same proportion. These components are stored in separate tanks. Both reservoirs feed two pumps in order to supply the required flow for optimum mixture.

Each electric pump is connected to a Variable Frequency Drive (VFD) which control their speeds. The pumped fluids are mixed with a mixing valve that gives the final bicomponent, which is used to make the wind generator blades. Due 
to the blade form, the output flow will vary depending on the part of the blade under creation. The process does not require a constant flow at the output. An example of the flow set point required is shown on Figure 1. Taken into account that the fluids are Non-Newtonian and the pumps efficiency are variable, the control system is not easy to carry out.

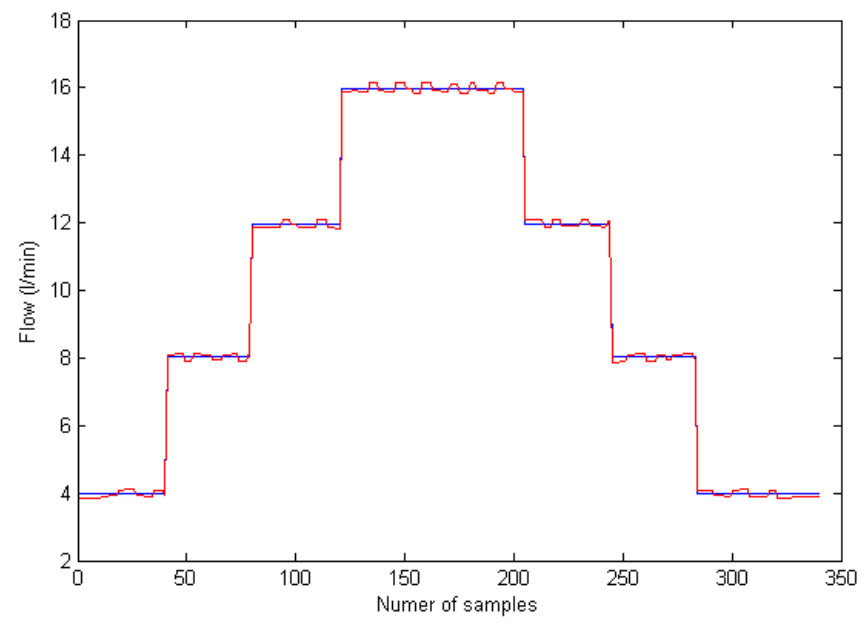

Fig. 1. Scheduled output flow rate

\subsection{System variables}

The dataset used on this research was obtained by measuring different system parameters during the plant operation. It is possible to measure the following variables of the system:

- Ratio set point: it represents the required mixture proportion. Its value is 1:1 always on this process.

- Real ratio: it represents real mixture proportion. It is one of the variables to be controlled.

- Flow rate set point: it is the desired flow rate at the output. It depends of the part of the blade under manufacture (cc/min).

- Flow rate $\mathrm{A}$, Flow rate $\mathrm{B}$ : they are the flow rate gave by pump $\mathrm{A}$ and $\mathrm{B}$ $(1 / \mathrm{min})$.

- Flow rate $\mathrm{A}+\mathrm{B}$ : it represents the sum of last two variables. It will vary depending of the flow rate set point value $(1 / \mathrm{min})$.

- Pump speed A, Pump speed B: they are the speeds of the pump A and B (rpm) respectively. 
- Pressure out pump A, Pressure out pump B: these variables represent the pressure at the output of the pump A and B (bar).

- Pressure out flow meter A, Pressure out flow meter B: they are the pressure measured after each flow meter (bar).

Firstly, it is necessary to classify these empirical data as inputs or outputs. In Figure 2 it is shown the system topology with the inputs and the outputs.

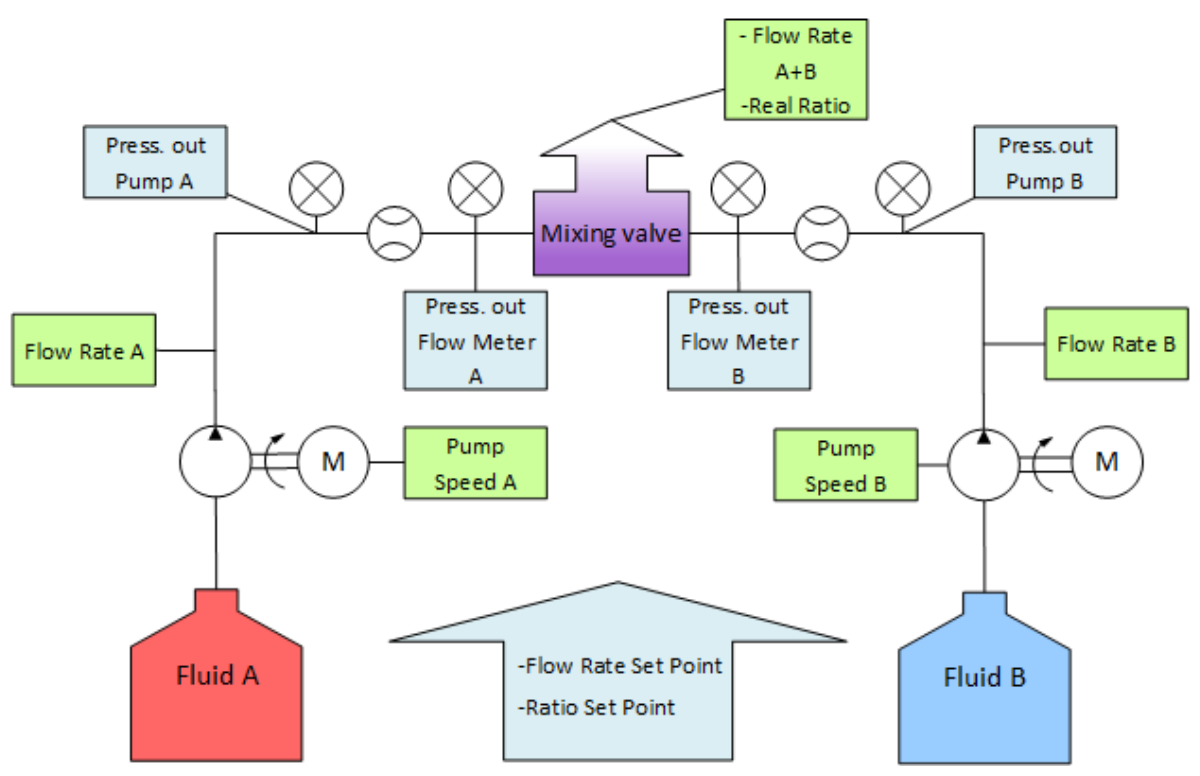

Fig. 2. Process layout

\section{Model approach}

As was mentioned above, global and local models have been taken into account with the aim to obtain a good approach performance. There is only one group for the case of global models, and there are several groups when local models are used. Therefore, it is necessary to apply regression techniques on each group. In both cases the modeling process is shown in Figure 3.

The Figure 4 shows in a graphical way the data classification process into $\mathrm{N}$ different clusters.

\subsection{Obtaining dataset}

The dataset used on this research was obtained by many different tests during the operation process. In Figure 5 it is possible to see the pump speed measured by 


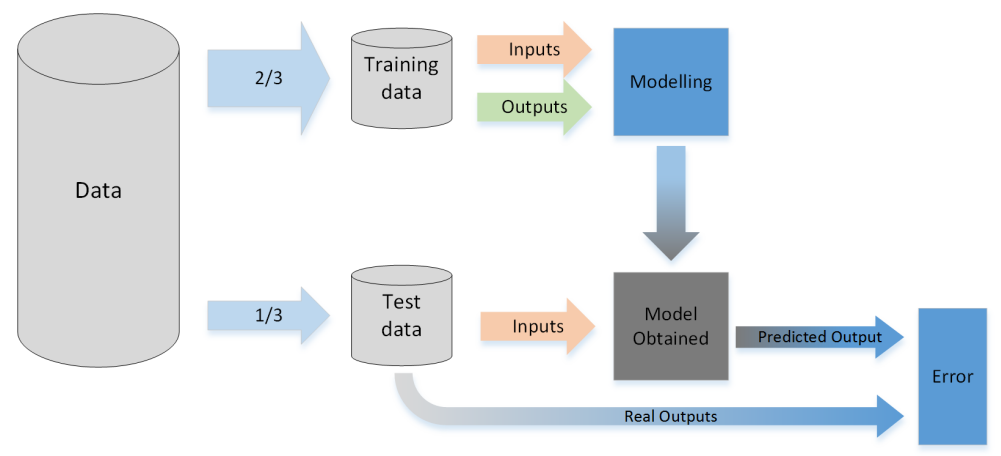

Fig. 3. Modeling process
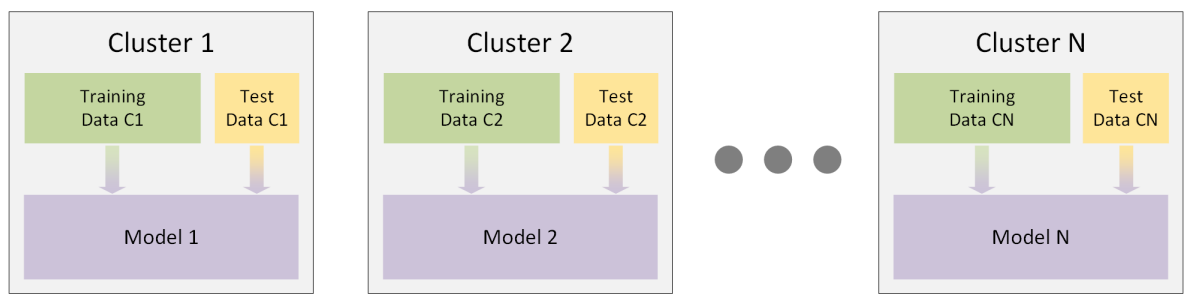

Fig. 4. Obtaining local models

a sensor while the system is working. This is one of the variables to be modeled. As can be seen in this figure, there are some mistakes on the data acquisition.

The dataset was conditioned by removing the wrong measures for a good modeling. After this task, our dataset was reduced from 9511 to 8549 samples.

\subsection{Techniques considered to create the model}

In this section are described different techniques used in order to obtain representative models of the system.

Self-Organization Map (SOM) Self-Organization Map [14] was created to visualize data behaviour on a low dimensional visualization. This technique uses unsupervised learning $[16,15]$. It is based on an array of nodes which are connected to $\mathrm{N}$ inputs by an $\mathrm{N}$-dimensional weight vector. The process is implemented by an iterative online algorithm but it is possible to be implemented by a batch version.

As a result of the process, SOM is expected to capture the geometry of the data. Thus, it is possible to visualize a $2 D$ representation. All process allows to obtain an idea of the number of the necessary clusters. 


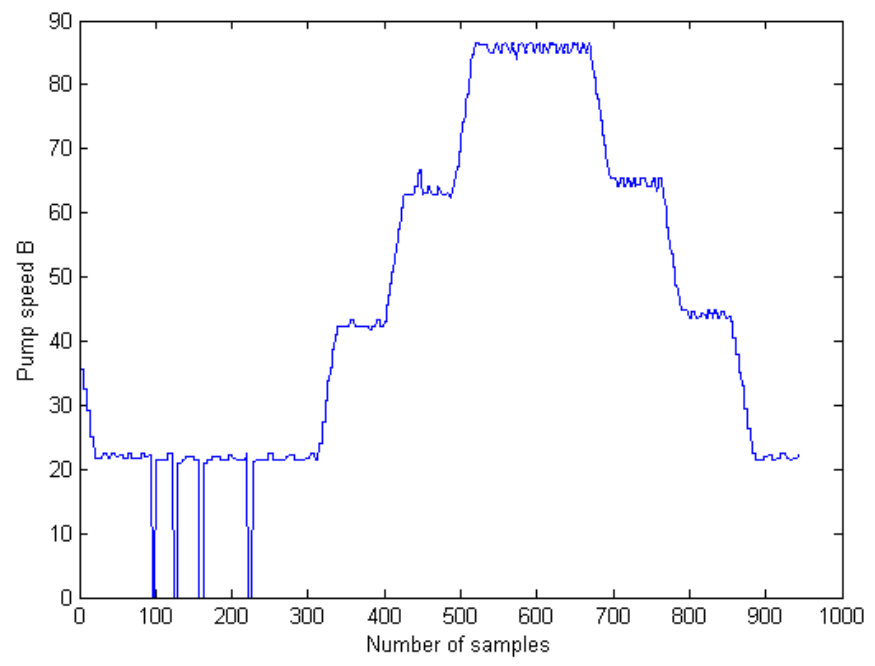

Fig. 5. Pump speed B, pure data

Data clustering. K-means algorithm Clustering algorithm organizes unlabeled feature vectors into different groups. Each group or cluster contain all data similar with similar features [13]. It is an unsupervised method of data grouping depending on the similarity [20,27]. Many different clustering algorithms have been used and new clustering algorithms continue to appear. Most of these algorithms are based on two clustering methods: agglomerative hierarchical clustering and iterative square-error partitional clustering [19]. The partitional clustering algorithms divide the data into a number of clusters trying to reduce the error function. The number of clusters is normally predefined, but it can be part of the error function $[7,23]$. The main purpose is to obtain a partition which, for a specific number of clusters, minimizes the square error.

The final grouping will depend of the number of clusters and on the initials clusters centers. The first one is more important because its prediction is not an easy task. K-means partitional clustering algorithm brings good results, over all, if the clusters are closed, well-separated in hyperspace and hyperspherical in shape. It is also able to detect hyper ellipsoidal-shaped clusters and it is computationally effective [7].

Artificial Neural Networks (ANN). Multi-Layer perceptron (MLP) A multilayer perceptron is a feedforward artificial neural network $[26,29]$. It is one of the most used ANNs due to its simple structure and its robustness. In spite of this fact, the ANN architecture must be selected properly in order to obtain good results. Multi-Layer Perceptron is composed by one input layer, one or more hidden layers and one output layer. Each layer has neurons, with an 
activation function. In a typical configuration, all neurons form a layer have the same activation function. This function could be a step, linear, log-sigmoid or tan-sigmoid.

Support Vector Regression (SVR), Least Square Support Vector Regression (LS-SVR) SVR is a modification of the Support Vector Machines (SVM) algorithm, used for classification. The main idea of the SVR is to map the data into a high-dimensional feature space $F$ by a non linear mapping and to do linear regression in this space [5,21].

The approximation of the solution is obtained by solving a linear equations system, and it is similar to SVM in generalization performance terms [22, 25]. The use of LS-SVM to regression is known as LS-SVR [9,24]. Its insensitive loss function is replaced by a squared loss function, that makes the Lagrangian by solving a linear KarusKuhn-Tucker KKT.

\subsection{Model selection}

After the model training, the Test data was used to calculate the MSE (Mean Squared Error) with the aim to select the best regression technique. The MSE of the global model was compared with the MSE for different number of clusters, to select the optimal configuration.

\section{Results}

The main objective is to select the best model that predicts the system behaviour.

First of all, there was a study based on SOM analysis to discover if correlation exists between different inputs, reducing the number of the inputs to the final model. The SOM analysis is used too to determine the optimal number of clusters, but, as is showing, the results are not as clear as it was supposed at first. Therefore, the data was divide into clusters with the K-means algorithm, and, for each cluster, different regression techniques were used, selecting the best techniques by MSE calculation.

\subsection{SOM analysis}

The neighbor distance, shown in Figure 6, defines the different zones of neurons in SOM structure. The clusters are separated by dark lines, and light areas on the map should correspond to a cluster. The SOM analysis is not definitely clear, and the selection of the number of clusters utilized is a subjective decision.

With the purpose of selection the optimal clusters, the dataset was divided in different number of clusters, from 5 to 10 , and the optimal number is selected after an MSE analysis of all procedure. All the results were compared with the MSE for global model. 


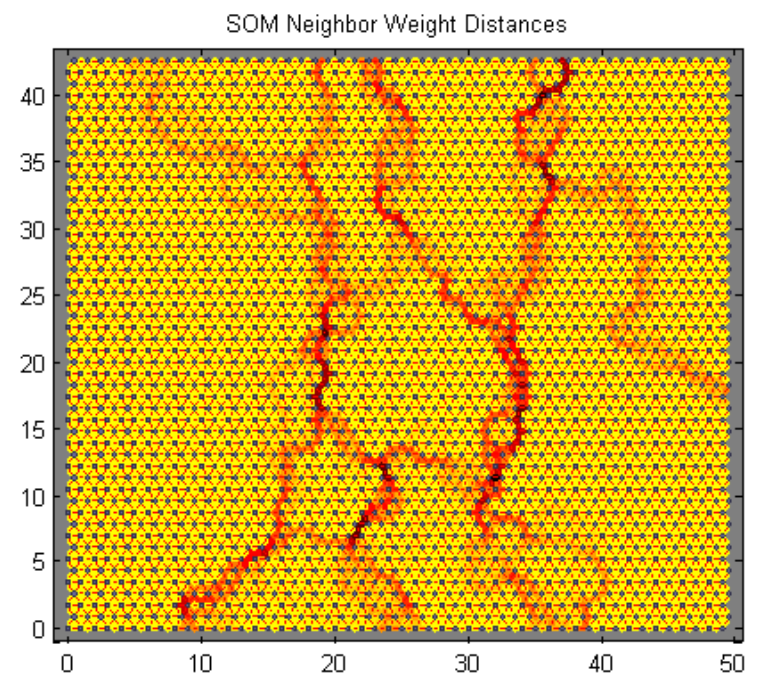

Fig. 6. SOM neighbor distances

The SOM input planes for each input of the SOM net, Figure 7, represent the reaction of the net to a specific input. In the figure, the Inputs 3 and 5 , the 'Press. out Pump A' and the 'Press. out Flow Meter A', have similar reactions. The same case occurs for Inputs 4 and 6 , the same variables for the B side of the system, it is meant that these inputs are correlated each other. The Input 1 , the 'Ratio Set Point', has always the same value, $1: 1$ for the bicomponent used in this research. In case of changing this bicomponent, the new 'Ratio Set Point' could be different, but always the same another time. The 'Input 2 - Flow Rate Set Point', is unique and different for the others inputs.

With the last explanation, at final, only three inputs are considered to create the model of the system; these inputs are:

- Input 2 - Flow Rate Set Point.

- Input 3 - Pressure out Pump A.

- Input 4 - Pressure out Pump B.

\subsection{Regression analysis}

As it was explained, the optimal number of clusters was decided after the calculation of the average of the MSE for all the outputs. For each cluster, the two regression techniques (ANN and SVR) were trained. The configuration of the SVR is an automatized task, but the ANN configuration should be selected properly. In this research, the number of neurons in the hidden layer of the network was varied from 3 to 20 . The activation function in these neurons was always tan-sigmoid, and the ouput layer neurons had a linear activation function. 

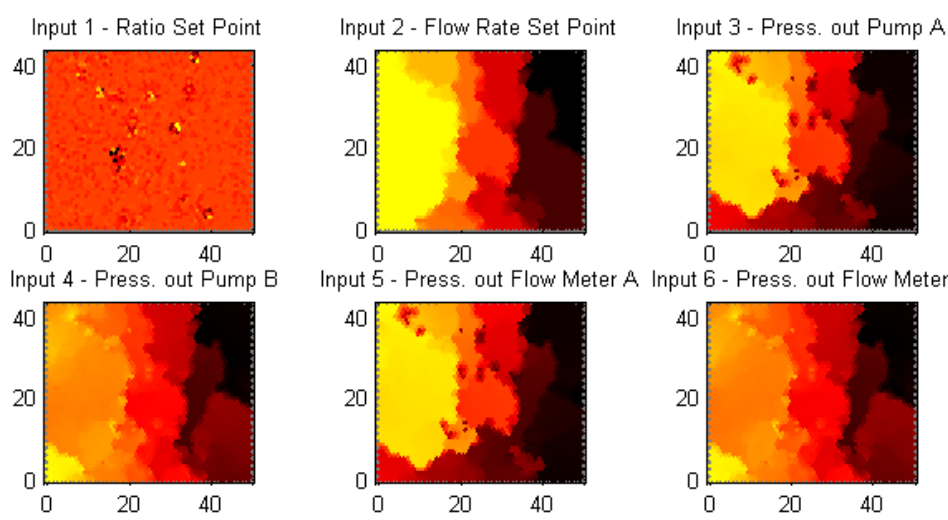

Fig. 7. SOM input planes

The best results for the ANN regression, with 5 neurons in the hidden layer, are shown in Table 1. In this table, the results for all the clusters configuration are presented. It is possible to see that the optimal clusters' number is 6 , based on the average MSE. However, if the number of clusters was different for each output, the model performance could be better, but the complexity would be increased more than the improvement achieved.

\begin{tabular}{|c||c|c|c|c|c|c||c|}
\hline & $\begin{array}{c}\text { Real } \\
\text { ratio }\end{array}$ & $\begin{array}{c}\text { Flow } \\
\text { rate A+B }\end{array}$ & $\begin{array}{c}\text { Flow } \\
\text { rate A }\end{array}$ & $\begin{array}{c}\text { Flow } \\
\text { rate B }\end{array}$ & $\begin{array}{c}\text { Pump } \\
\text { speed A }\end{array}$ & $\begin{array}{c}\text { Pump } \\
\text { speed B }\end{array}$ & Average \\
\hline \hline Global model & 0.52260 & 0.42362 & 0.10503 & 0.10373 & 0.73050 & 0.84887 & 0.45572 \\
\hline 5 clusters & 0.51698 & 0.37985 & 0.09366 & 0.09258 & 0.59531 & 0.74568 & 0.40401 \\
\hline 6 clusters & 0.42997 & 0.38097 & 0.09393 & 0.09282 & 0.56373 & 0.71347 & 0.37915 \\
\hline 7 clusters & 0.47842 & 0.31058 & 0.07597 & 0.07511 & 0.61409 & 0.79754 & 0.39195 \\
\hline 8 clusters & 0.43980 & 0.45649 & 0.11283 & 0.11088 & 0.96655 & 1.26514 & 0.55891 \\
\hline 9 clusters & 1.27257 & 0.46047 & 0.11423 & 0.11258 & 0.59370 & 0.77710 & 0.55511 \\
\hline 10 clusters & 0.42680 & 0.38379 & 0.09449 & 0.09315 & 0.78581 & 0.96995 & 0.45900 \\
\hline
\end{tabular}

Table 1. MSE obtained using ANN

The results for the SVR regression are shown in Table 2. In this case, the optimal separation of dataset is 9 . However, with the SVR occurs the same as before, if the number of clusters was different for each output, the MSE could be better.

\section{Conclusions}

This research provides an accurate method of modeling a bicomponent mixing system, used in wind generator blades manufacturing. 


\begin{tabular}{|c||c|c|c|c|c|c||c|}
\hline & $\begin{array}{c}\text { Real } \\
\text { ratio }\end{array}$ & $\begin{array}{c}\text { Flow } \\
\text { rate A+B }\end{array}$ & $\begin{array}{c}\text { Flow } \\
\text { rate A }\end{array}$ & $\begin{array}{c}\text { Flow } \\
\text { rate B }\end{array}$ & $\begin{array}{c}\text { Pump } \\
\text { speed A }\end{array}$ & $\begin{array}{c}\text { Pump } \\
\text { speed B }\end{array}$ & Average \\
\hline \hline 5 clusters & 0.15511 & 0.30999 & 0.07228 & 0.07466 & 0.36859 & 0.41285 & 0.23225 \\
\hline 6 clusters & 0.15494 & 0.23023 & 0.06358 & 0.05983 & 0.42614 & 0.63239 & 0.26118 \\
\hline 7 clusters & 0.16321 & 0.29375 & 0.07305 & 0.07254 & 0.38717 & 0.47223 & 0.24366 \\
\hline 8 clusters & 0.19468 & 7.34780 & 1.85414 & 1.82709 & 255.51247 & 451.48554 & 119.70362 \\
\hline 9 clusters & 0.16087 & 0.23890 & 0.06001 & 0.06163 & 0.32868 & 0.41169 & 0.21030 \\
\hline 10 clusters & 0.15778 & 0.22213 & 0.05443 & 0.06473 & 0.31153 & 1.14401 & 0.32577 \\
\hline
\end{tabular}

Table 2. MSE obtained using SVR

The achieved model predicts the behaviour of the real system when the inputs are known. This model has been obtained from an empirical dataset. This model approach is based on a hybrid intelligent system by combining different regression techniques on local models.

After several tests, the analysis of the results shows that the best model configuration has 9 clusters. The regression technique employed on each one was SVR. The best average MSE obtained with this configuration was 0.2103.

This kind of analysis could be applied to many different systems with the aim to improve several goals like: efficiency, performance, features of the obtained material, and so on.

Remark that the final hybrid model achieves good results although the system has an important nonlinear nature.

\section{Acknowledgments}

This research has been funded by the University of León, and by the Spanish Ministry of Education, Culture and Sport (grant for Collaboration in University Departments).

\section{References}

1. Alvarez-Huerta, A., Gonzalez-Miguelez, R., García-Metola, D., Noriega-Gonzalez, A.: Drywell tempeture prediction of a nuclear power plant by means of artificial neural networks. Dyna 86(4), 467-473 (2011)

2. Bishop, C.: Pattern recognition and machine learning (information science and statistics). Springer-Verlag New York, Inc., Secaucus, NJ, USA (2006)

3. Calvo-Rolle, J., Casteleiro-Roca, J., Quintián, H., Meizoso-Lopez, M.: A hybrid intelligent system for PID controller using in a steel rolling process. Expert Systems with Applications 40(13), 5188-5196 (2013)

4. Cherif, A., Cardot, H., Boné, R.: SOM time series clustering and prediction with recurrent neural networks. Neurocomput. 74(11), 1936-1944 (may 2011)

5. Cristianini, N., Shawe-Taylor, J.: An introduction to support Vector Machines and other kernel-based learning methods. Cambridge University Press, New York, NY, USA (2000) 
6. Fan, H., Wong, C., Yuen, M.F.: Prediction of material properties of epoxy materials using molecular dynamic simulation. In: Thermal, Mechanical and Multiphysics Simulation and Experiments in Micro-Electronics and Micro-Systems, 2006. EuroSime 2006. 7th International Conference on. pp. 1-4 (April 2006)

7. Garg, L., Mcclean, S., Meenan, B., Millard, P.: Phase-type aurvival trees and mixed distribution survival trees for clustering patients' hospital length of stay. Informatica $22(1), 57-72($ jan 2011)

8. Ghaseminezhad, M.H., Karami, A.: A novel self-organizing map (SOM) neural network for discrete groups of data clustering. Appl. Soft Comput. 11(4), 37713778 (jun 2011)

9. Guo, Y., Li, X., Bai, G., Ma, J.: Time series prediction method based on LS-SVR with modified gaussian RBF. In: Huang, T., Zeng, Z., Li, C., Leung, C. (eds.) Neural Information Processing, Lecture Notes in Computer Science, vol. 7664, pp. 9-17. Springer Berlin Heidelberg (2012)

10. Jacobs, R., Jordan, M., Nowlan, S., Hinton, G.: Adaptive mixtures of local experts. Neural Comput. 3(1), 79-87 (mar 1991)

11. Jordan, M., Jacobs, R.: Hierarchical mixtures of experts and the EM algorithm. Neural Comput. 6(2), 181-214 (mar 1994)

12. Karasuyama, M., Nakano, R.: Optimizing svr hyperparameters via fast crossvalidation using aosvr. In: Neural Networks, 2007. IJCNN 2007. International Joint Conference on. pp. 1186-1191 (Aug 2007)

13. Kaski, S., Sinkkonen, J., Klami, A.: Discriminative clustering. Neurocomputing 69(13), 18-41 (2005)

14. Kohonen, T.: The self-organizing map. Proceedings of the IEEE 78(9), 1464-1480 (1990)

15. Kohonen, T.: Exploration of very large databases by self-organizing maps. In: Neural Networks,1997., International Conference on. vol. 1, pp. PL1-PL6 vol.1 (1997)

16. Kohonen, T., Oja, E., Simula, O., Visa, A., Kangas, J.: Engineering applications of the self-organizing map. Proceedings of the IEEE 84(10), 1358-1384 (1996)

17. Li, H., Chen, Z.: Overview of different wind generator systems and their comparisons. Renewable Power Generation, IET 2(2), 123-138 (June 2008)

18. Martínez-Rego, D., Fontenla-Romero, O., Alonso-Betanzos, A.: Efficiency of local models ensembles for time series prediction. Expert Syst. Appl. 38(6), 6884-6894 (Jun 2011)

19. Pal, N., Biswas, J.: Cluster validation using graph theoretic concepts. Pattern Recognition 30(6), 847-857 (1997)

20. Qin, A., Suganthan, P.: Enhanced neural gas network for prototype-based clustering. Pattern Recogn. 38(8), 1275-1288 (aug 2005)

21. Steinwart, I., Christmann, A.: Support vector machines. Springer Publishing Company, Incorporated, 1st edn. (2008)

22. Suykens, J., Vandewalle, J.: Least squares support vector machine slassifiers. Neural Processing Letters 9(3), 293-300 (1999)

23. Šutienè, K., Makackas, D., Pranevičius, H.: Multistage k-means clustering for scenario tree construction. Informatica 21(1), 123-138 (jan 2010)

24. Wang, L., Wu, J.: Neural network ensemble model using PPR and LS-SVR for stock market eorecasting. In: Huang, D.S., Gan, Y., Bevilacqua, V., Figueroa, J. (eds.) Advanced Intelligent Computing, Lecture Notes in Computer Science, vol. 6838, pp. 1-8. Springer Berlin Heidelberg (2012)

25. Wang, R., Wang, A., Song, Q.: Research on the alkalinity of sintering process based on LS-SVM algorithms. In: Jin, D., Lin, S. (eds.) Advances in Computer Science 
and Information Engineering, Advances in Intelligent and Soft Computing, vol. 168, pp. 449-454. Springer Berlin Heidelberg (2012)

26. Wasserman, P.: Advanced methods in neural computing. John Wiley \& Sons, Inc., New York, NY, USA, 1st edn. (1993)

27. Ye, J., Xiong, T.: Svm versus least squares SVM. Journal of Machine Learning Research - Proceedings Track 2, 644-651 (2007)

28. Young, W.B., Wu, W.H.: Optimization of the skin thickness distribution in the composite wind turbine blade. In: Fluid Power and Mechatronics (FPM), 2011 International Conference on. pp. 62-66 (Aug 2011)

29. Zeng, Z., Wang, J.: Advances in neural network research and applications. Springer Publishing Company, Incorporated, 1st edn. (2010)

30. Zuo, Y., Liu, H.: Evaluation on comprehensive benefit of wind power generation and utilization of wind energy. In: Software Engineering and Service Science (ICSESS), 2012 IEEE 3rd International Conference on. pp. 635-638 (June 2012) 